\title{
Product Modelling and Rationale Capture in Design Process
}

\author{
J.P.T. Mo \\ CSIRO Manufacturing Science and Technology, Australia \\ Em:John.Mo@cmst.csiro.au
}

Keywords Product Modelling, Design Rationale, Knowledge Capture, Global Design

\begin{abstract}
Product design is an intuitive activity and generates a lot of information supporting the product in the rest of its life cycle. These information consist of both foreground (explicitly recorded) and background (normally unconsciously referred in practice) information. This paper describes a system developed based on the hierarchical structure of product modelling methodology for capturing an important part of the un-written information, design rationale. Developed in a Java environment, the system is platform independent and is designed to be extensible into a global design support system via the internet.
\end{abstract}

\section{INTRODUCTION}

In recent years, the philosophy of global manufacturing has been developed among aggressive manufacturers who are prepared to take on the challenge of managing their activities in the most effective fashions in different parts of the world [1]. More specifically, product development is an essential operation involving business partners and customers geographically distributed [2]. During the design phase, designers need to cooperate in all aspects of the design. With the help of the latest CAD technologies, design teams are using various kinds of product models to represent their products. STEP and other international standards have helped to foster information transfer among different players [3].

However, designers in different parts of the world have the problem of communicating their design idea to their colleagues elsewhere when they distribute their CAD models. Information represented by CAD models are primarily explicit information of the product. The reasoning behind the design are often forgotten when the product model is distributed to the other 
members of the design team. In other words, the intent (or rationale) of the feature or details of the design is not recorded due to lack of such facility.

The system described in this paper is a Java based design intent capture and management system which captures the design intent (what the design thinks) along with normal design information. The system uses the hierarchical structure of product modelling methodology and provides an active tool during the design process to help recording the rationale information from the designer.

\section{GLOBAL INFORMATION MANAGEMENT SYSTEM}

The purpose for the Global Information Management System (GIM) is to cater for the need of non-interactive sessions between 2 or more geographically separated design groups in different time zones. While the use of tele-presence tools such as video conferencing is expected to provide an interactive environment for several design groups to work together in a specific time window to communicate, this only occurs in a short period within the 24 hour clock product development cycle [4]. In the other time periods, only people in one time zone will be on-line and all the others will take their rest.

The research to generate the Global Information Management System is focussed on the issue of asynchronous access to information. In a global concurrent engineering situation, design teams will be spread across different time zones, and will thus need to access information from other sites when they are off-line, or will need to know what happened at others site when they themselves were off-line.

The system is aimed to provide support to the on-line design group to search, retrieve, view, edit, be alerted, be managed and more importantly, capture design rationale. To do these, an understanding of the system architecture and elements required to build such a system is required [5]. It is based on an object oriented object structure of the product and fits the implicit information of the product into relevant locations of the data structure. By definition, explicit information are those related to the design of the product such as user specification, material, overall dimensions and functions. These are captured by CAD systems. Implicit information are those information not normally expressed explicitly by the design. Typical examples are the reasons for certain material selection or the size of some mating features. These information are captured through a series of windows linked to the product data model so that the rationale behind the designed features can be recorded accordingly. 


\section{METHODOLOGY OF SYSTEM DESIGN}

The methodology applied to develop the system is to treat all design activities as activities manipulating design objects. The definition of design objects originates from the concept of data abstraction for compute aided process planning [6]. There are three levels of data abstraction in the framework of objects which is required to develop a unified system linking CAD model data to manufacturing. It makes use of a universally acceptable set of objects which can interact with process planning modules (which are themselves objects) to achieve the required output to manufacturing. With this approach, a part is composed of a series of objects which possess certain uniquely identifiable characteristics. As the design process progresses, the objects are moved to higher levels where more attributes are added or inherited from other objects.

In the design of GIM, the relationships of the objects are taken into account. In most cases, the cardinality of the object relationships are multiples (n-to-m). When the relationships are defined in the product model, object methods can be developed to pop-up windows (e.g. to alert designer of conflicts) through which the designer can enter information relevant to that relation. This situation can be illustrated in Figure 1.

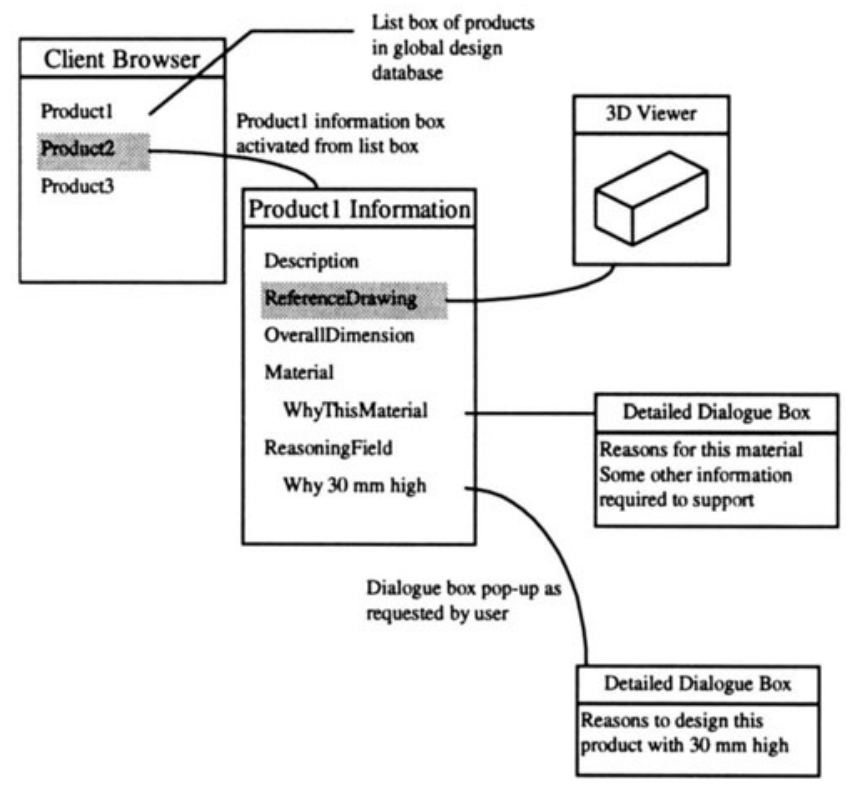

Figure 1 System User Interaction Scenario 


\section{AN EXAMPLE PRODUCT - DINING TABLE}

The design rationale information has to be recorded in a form which is easily retrieved and displayed. One important characteristics of this database is that it must be object oriented. Figure 2 shows the outcome of a product model illustrating some of the basic ideas in OOM of product. It describes the relationships and attributes of the design of a "Dining Table" which is formed by packing a table and a table cloth as a product.

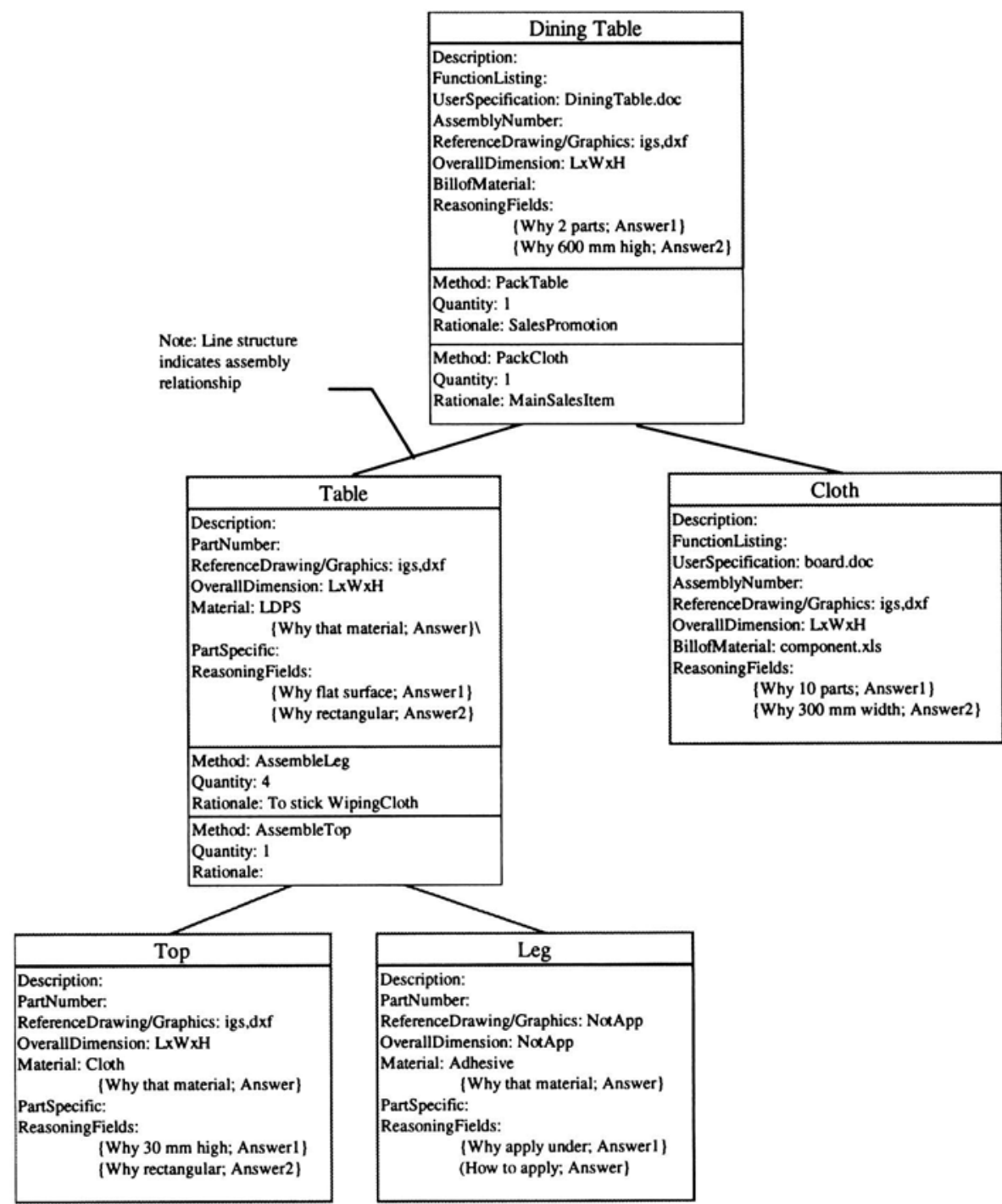

Figure 2 The Dining Table Product Model 


\section{IMPLEMENTATION}

Using the tree objects in Java, the product model structure can be implemented as shown in Figure 3. Assembly objects are given the same set of attributes. At the top level, the dining table is the root of the tree. Description information relevant to that assembly is displayed when the level is highlighted.

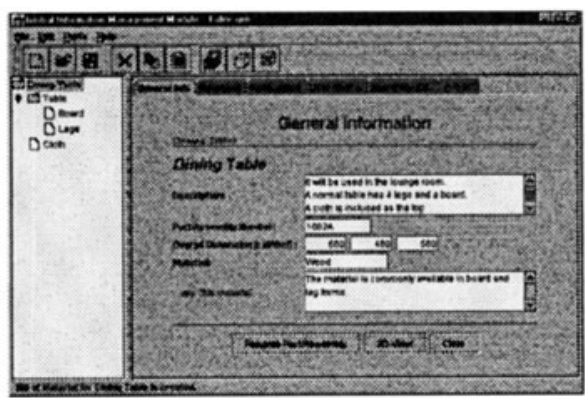

(a)

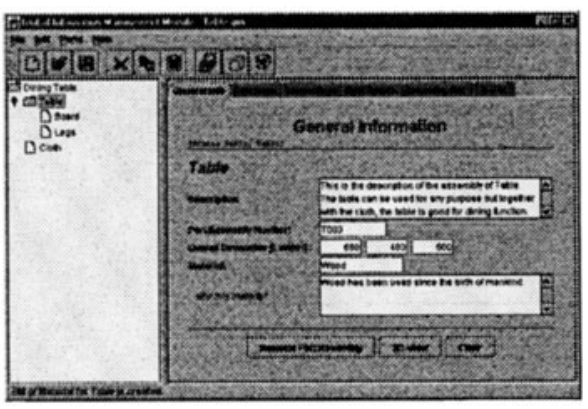

(b)

Figure 3 Top level model information screens

A product has functions to perform. The important implicit information of a product are not usually recorded in a CAD environment. These are captured in the other tabbed-panes. The "Function Listing" screen (Figure 4) captures the function of the object or assembly. Reasons for various aspects of the design (can be totally unrelated to CAD) are captured in a freely entered screen. By highlighting the reasoning question in the list, the answers are captured and displayed at the bottom window (Figure 5).

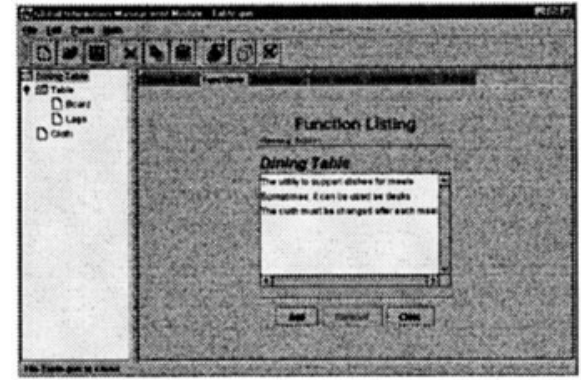

Figure 4 Function Listing

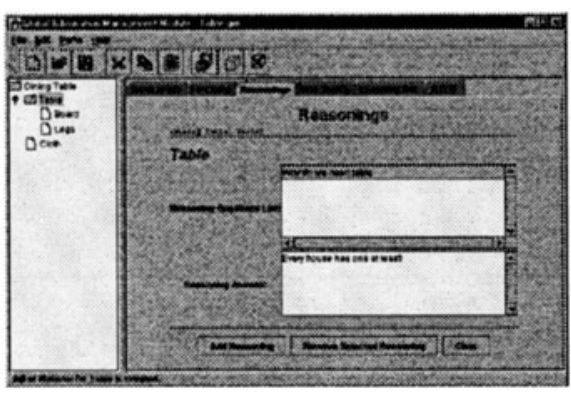

Figure 5 Reasoning capture

To help the designer, a simple product viewing capability is also included (Figure 6). It is planed that the viewer will be integrated with a 
global CAD system developed in a complementary project later [7]. Customer information are usually supplied in a wide variety of format. At this stage of development, a HTML browser is included (Figure 7). Browsers of other formats can be incorporated into the system easily.

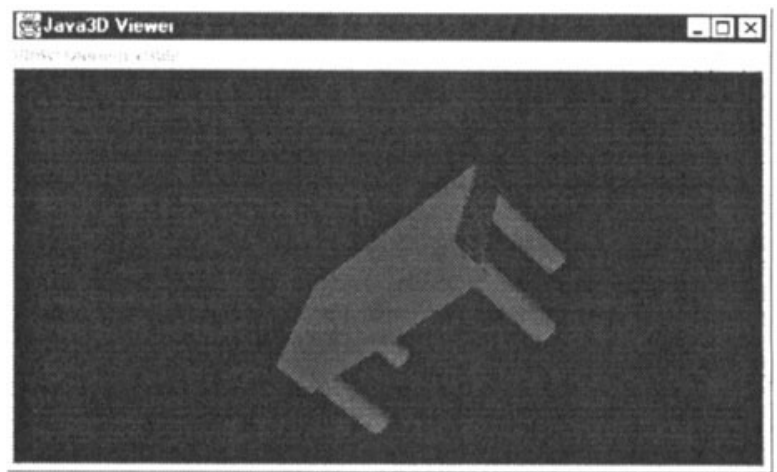

Figure $63 D$ product viewing window

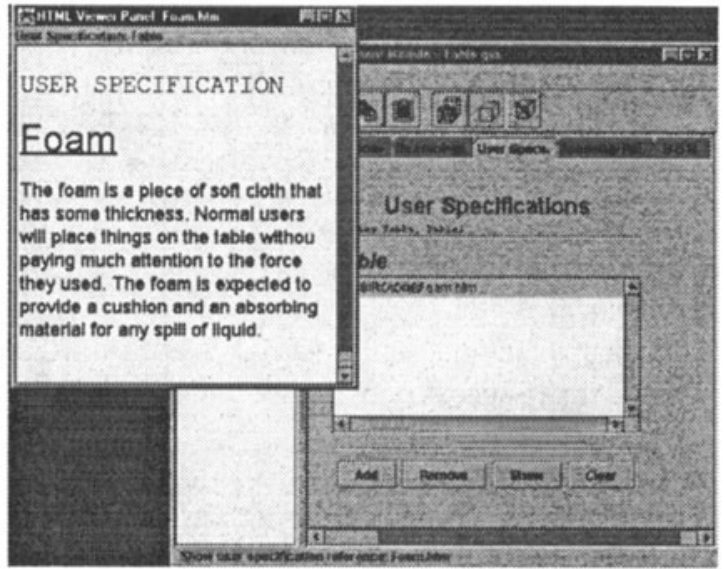

Figure 7 Customer specification

\section{CONCLUSION}

A system for capturing design rationale has been created using an object oriented product modelling structure and the internet based programming language, Java. Developed in an object oriented environment, the system captures explicit information of the design as well as implicit information of the product according to the level of the object in the model. Further work is planned to expand the capability to interface to CAD systems in a more dynamic way. It includes the design and implementation of an enhanced system architecture which interacts with the user while he/she is working on 
the design. For example, an automatic pop up query based on the product model structure will appear to capture the intent of the designer at the time when the designer is making the drawing. These features can be done readily with the product modelling background of GIM.

\section{REFERENCES}

[1] Redman J., Mo J.P.T. (1999). "Process Modelling for Global Work Team Creation and Management”, 2nd International Conference, Managing Enterprises '99, 18-20 November, Newcastle, Australia, pp.275-280

[2] Shinonome M., Hashimoto H., Fuse A., Mo J.P.T. (1998). "Development of an information technology infrastructure for extended enterprise", ", IFIP TC5 WG5.3/5.7 Third International Conference on the Design of Information Infrastructure Systems for Manufacturing (DIISM '98), Paper 23, May 18-20, Fort Worth, Texas, U.S.A., pp.353364

[3] Chen Y.M., Hsiao Y.T. (1997). "A collaborative data management framework for concurrent product and process development", International Journal of Computer Integrated Manufacturing, Vol.10, No.6, pp.446-469

[4] Mo J.P.T., Kovacek M., Cirocoo L (1998). "A Framework for Round the clock Design and Support", Proceedings of the $10^{\text {th }}$ International IFIP WG5.2/5.3 International Conference PROLAMAT 98,.9-12 September, Trento, Italy, pp.439-450

[5] Ooi S.T., Bertok P., Zhao L.P., Jiang H.C., Mo J.P.T. (1999). "Patterns as Reengineering Front-End in Distributed Environments", Proceedings of $2^{\text {nd }}$ Australian Workshop on Software Architectures, 23 November, Melbourne, Australia, pp.75-82

[6] Mo J.P.T., Ranganathan D. (1996). "From Design to Manufacture by Objects", International Journal of Flexible Automation and Integrated Manufacturing, Vol.4, No.2, 1996, pp.95-110

[7] Jiang H.C., Mo. J.P.T. (1999). "Internet Based Design System for Global Concurrent Engineering”, 2nd International Conference, Managing Enterprises '99, 18-20 November, Newcastle, Australia, pp.150-156 